\title{
Microfluidique et optofluidique
}

Yong CHEN et Géraldine HALLAIS - École Normale Supérieure, 24 rue Lhomond, 75005 Paris

yong.chen@ens.fr

Les concepts de miniaturisation et d'intégration développés par l'industrie de la microélectronique ont connu un essor considérable dans plusieurs autres domaines non seulement pour la fabrication d'éléments mécanoélectriques, optiques et magnétiques mais aussi pour la réalisation de véritables laboratoires de biologie ou de chimie sur puce. Ces derniers sont basés sur la manipulation de fluide en très faible volume afin de contrôler de façon précise et efficace des réactions (bio)chimiques - d'où l'émergence d'une nouvelle discipline, la microfluidique. Déjà implantée dans de nombreux domaines scientifiques tels que la biologie, la chimie, la physique, la médecine, etc., la microfluidique s'est aussi associée à l'optique et/ou la photonique pour développer de nouvelles fonctionnalités sur puce. L'objectif de cet article est $d^{\prime}$ introduire les notions de la microfluidique et $d^{\prime}$ expliquer comment l'optique peut y intervenir en donnant quelques exemples $d^{\prime}$ applications des puces optofluidiques.

\section{La microfluidique}

La microfluidique concerne avant tout la mise en mouvement et la gestion des écoulements de fluides de très faible volume $\left(10^{-9}\right.$ à $10^{-18}$ litres) dans des microcanaux, ainsi que les différentes opérations effectuées sur ces fluides comme mélange, séparation, contrôle de température... Ces fonctions sont implémentées par une variété de composants microfluidiques tels que des micropompes, des micro-vannes, des micromélangeurs, etc., tous intégrés sur une même puce. La microfluidique permet ainsi de réaliser des réactions chimiques ou biochimiques de haute précision et/ou à haut débit souvent difficiles à mettre au point macroscopiquement, ce qui a un intérêt majeur pour la recherche biomédicale, pharmaceutique, physicochimique, etc.

\section{Actionner les fluides}

L'actionnement des fluides dans un microcanal est en général assuré par une différence de pression entre les deux extrémités du canal générée par une pression hydrostatique, un pousse-seringue ou une pompe péristaltique. Ce type de solution est relativement simple mais le contrôle du débit doit être réalisé avec des connectiques assez encombrantes. Par ailleurs, il en résulte un écoulement qui, du fait des frottements sur les parois dus à la viscosité, est de type Poiseuille : il présente une distribution de vitesse parabolique à travers le canal, ce qui n'est pas désirable pour certaines applications telles que la séparation de biomolécules. Une solution consiste à appliquer un champ électrique le long du canal. Comme le fluide en mouvement est souvent une solution aqueuse chargée de différents sels, son contact avec les parois du canal crée des charges statiques à sa surface qui est équilibrée localement dans la solution par une couche de charges opposées d'une dizaine à une centaine de nanomètres d'épaisseur. L'interaction du champ électrique avec cette couche périphérique provoque sa mise en mouvement, ce qui entraîne le déplacement de l'ensemble du fluide (effet électro-osmotique) avec un profil de vitesse quasiment homogène à travers le canal (figure 1).
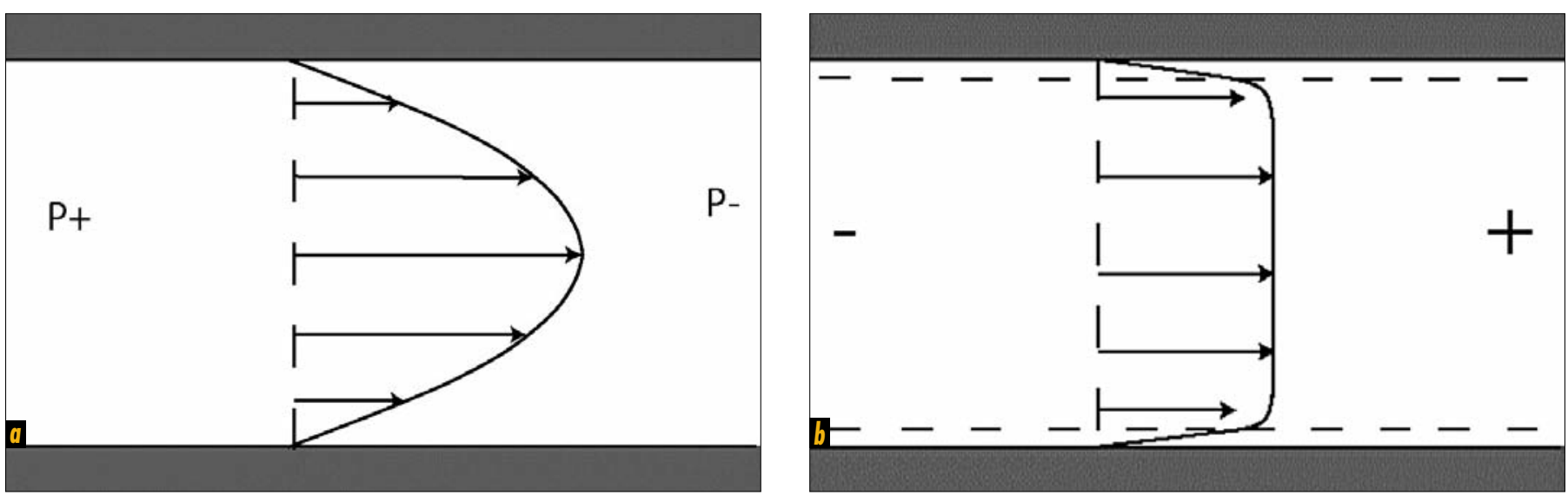

Figure 1. Profil de vitesse dans le cas d'un écoulement sous pression (a) et sous champ effet électro-osmotique (b). 


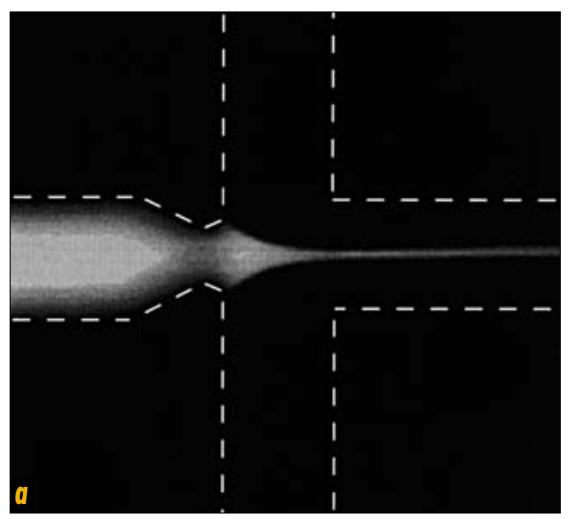

Figure 2. Écoulement laminaire dans un microcanal : (a) focalisation hydrodynamique (cf. J. Knight et al., Physical Review Letters, 1998) ; (b) mélange avec des microstructures de surface gravées (cf. A. Strook et al., Science, 2002).

Le cas des mélanges fluides

Lorsque plusieurs fluides sont injectés dans un canal de parois lisses, les écoulements sont laminaires en raison de la prépondérance des forces de viscosité ce qui se traduit par un petit nombre de Reynolds (rapport entre les forces d'inertie et les forces de viscosité). II est donc facile d'obtenir une focalisation hydrodynamique dans un canal en encadrant un fluide d'indice de viscosité élevé par un fluide d'indice plus faible. En revanche, il est plus difficile d'effectuer un mélange rapide de ces deux fluides. Une solution

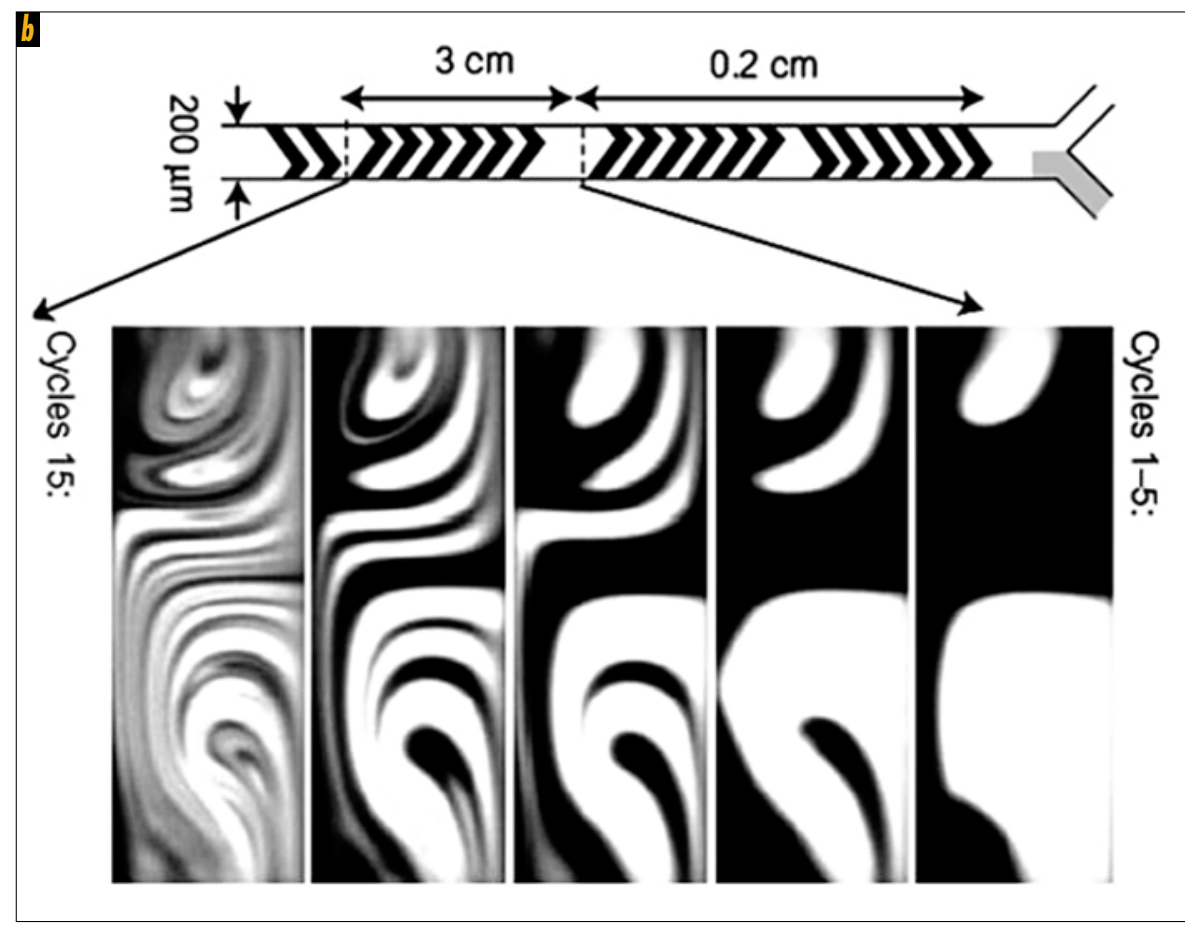

est de graver dans les parois planes du canal des microsillons avec une géométrie en dents de scie conduisant à l'apparition de turbulences (figure 2).

Lorsque deux fluides non miscibles sont injectés dans un canal, l'écoulement devient diphasique. En particulier, une chaine de micro-gouttes peut être produite par une injection des deux fluides dans un dispositif en configuration T (figure 3a) ou par la méthode de focalisation hydro- dynamique (dans la limite de son instabilité) (figure 3b). Une fois produites, les microgouttes peuvent être facilement manipulées (fission, fusion, etc.) en utilisant des micro-canaux de bifurcation, I'action d'un champ électrostatique, etc.

Les techniques de fabrication

La fabrication des puces microfluidiques fait appel à plusieurs filières technologiques.

\section{SPECTROGON}

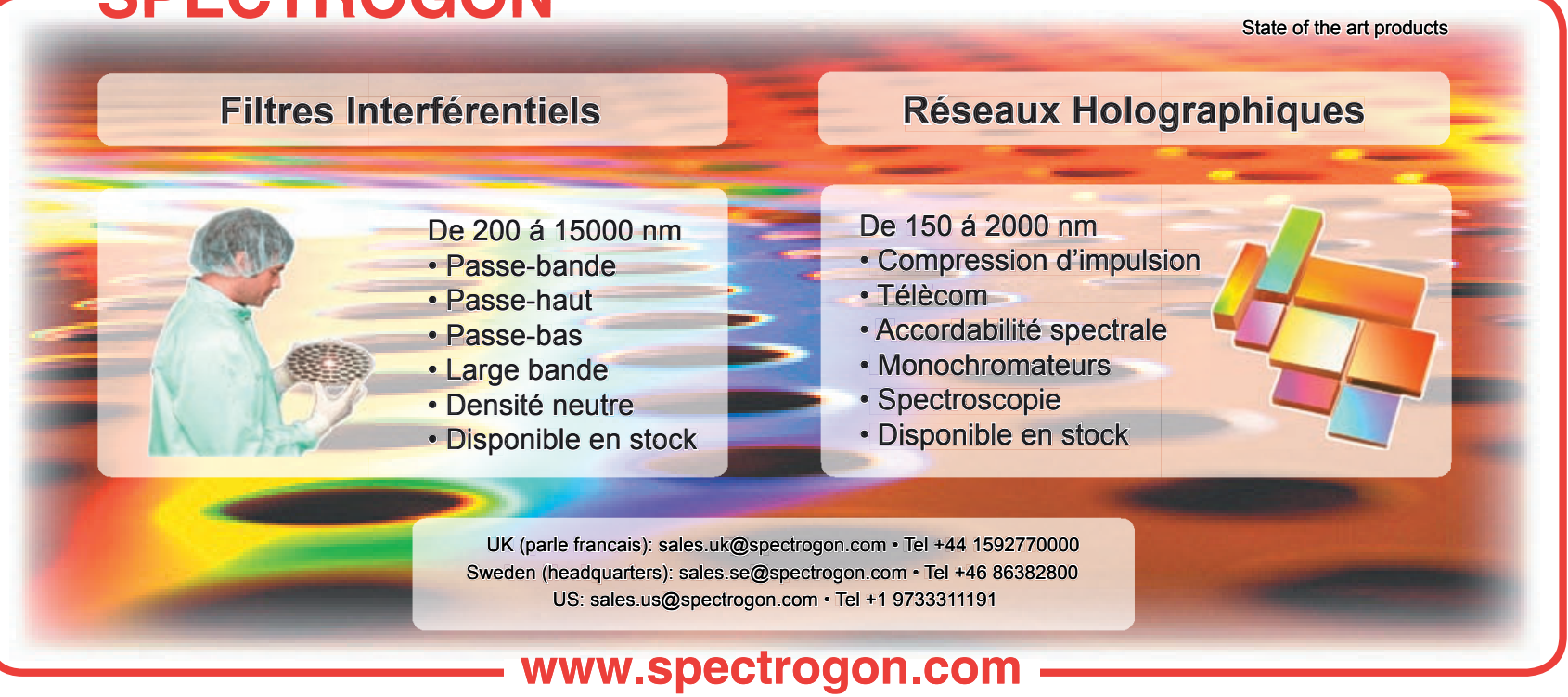



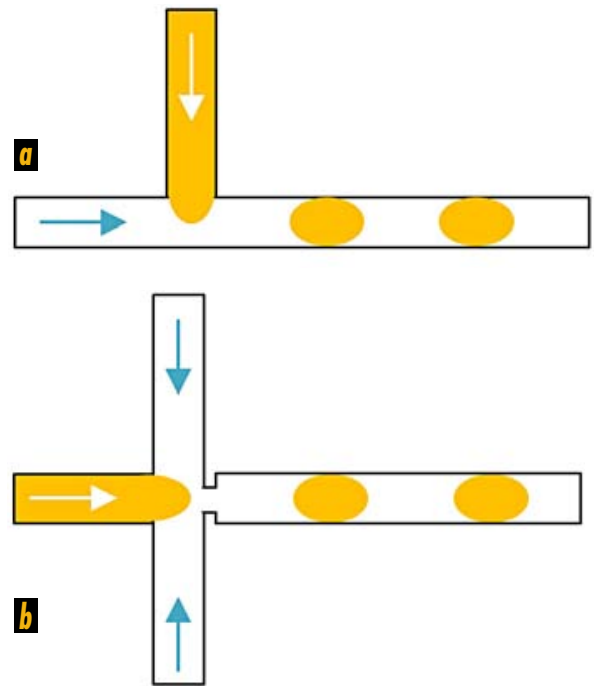

Figure 3. Production de micro-gouttes par jonction $T$ (a) et focalisation hydrodynamique (b).

La première est celle du silicium, issue du domaine de la microélectronique. Déjà largement exploitée pour la production des microsystèmes électromécaniques (MEMS), cette filière reste relativement peu adaptée à la microfluidique pour les laboratoires de recherche à cause de la complexité de ses procédés de fabrication et de la grande variété de dispositifs microfluidiques

à réaliser.

La deuxième filière est celle pour la production de dispositifs en verre. Si elle est déjà plus compatible avec les expériences en chimie et en biologie, il est cependant difficile d'intégrer des micro-vannes et des micro-pompes dans un dispositif tout en verre.

La troisième filière utilise des polymères, ce qui facilite la production de dispositifs en masse lpar moulage, injection, impression, etc.). En revanche, cette option est aussi limitée par l'absence de solutions techniques efficaces pour l'intégration de fonctionnalités diverses et variées.

Enfin, la quatrième filière est celle qui utilise l'élastomère de polydiméthylsi- loxane (PDMS). Basée sur la lithographie douce, elle occupe une place particulièrement importante dans les laboratoires de recherche, puisqu'il est très facile de mettre en œuvre un prototypage rapide et «bas coût» de puces ayant des fonctionnalités assez complexes. Le PDMS est transparent dans le domaine visible, ce qui facilite aussi les applications optiques. La figure 4 montre trois exemples de dispositifs microfluidiques réalisés au sein des laboratoires CNRS à Marcoussis (LPN, UPR2O) et à Paris (ENS Ulm, UMR8640) : un micro-réacteur en anneau avec des micro-vannes et des micropompes de contrôle intégrées, un microlaser à colorant avec des fibres optiques intégrées pour des mesures d'absorption intra-cavité, et un dispositif avec des multiélectrodes pour étudier des problèmes de stress oxydatif cellulaire.

\section{L'optofluidique et les applications optofluidiques}

L'intérêt de l'union entre la microfluidique et l'optique est de bénéficier à la fois du potentiel de l'optique et de celui de la microfluidique. En 2002, nous avons prouvé que l'intégration d'optique dans une puce microfluidique était possible en réalisant des guides d'ondes [1]. Ceséléments sont basés sur l'écoulement laminaire d'un fluide d'indice de réfraction élevé (huile - n 1.5) emprisonné dans un micro-canal structuré, lui, dans un matériau d'indice plus faible (PDMS n 1.4) couplé à une fibre optique clivée. Les phénomènes de prismes et de lentilles ont été observés de la même manière.

Grâce à la souplesse offerte par les technologies de la microfluidique, les propriétés de ces éléments peuvent être facilement modulées.

En parallèle, le groupe Whitesides à Harvard a conçu des guides d'onde en encadrant un fluide d'indice de réfraction élevé par un fluide d'indice plus faible et en les aiguillant dans tel ou tel canal (figure 5). Non seulement ce type de guide d'onde est configurable mais il présente une meilleure qualité d'interface entre le coeur et la gaine. Par conséquent, plusieurs autres types de réalisations ont été basés sur le concept de guidage modulable en changeant le milieu de travail comme des réfractomètres avec des réseaux de diffraction et des interféromètres de Mach-Zender sur puce microfluidique. Les propriétés optiques de ces éléments étant très sensibles au milieu de travail, ils permettent une mesure très précise de l'indice de réfraction du milieu.

Figure 4. Photographies d'un dispositif microfluidique avec un mélangeur en anneau activé par une pompe péristaltique intégrée (a), un microlaser à colorant avec plusieurs entrées de solutions à analyser (b) et un dispositif avec des microélectrodes intégrées pour I'analyse par voie électrochimique (c). 

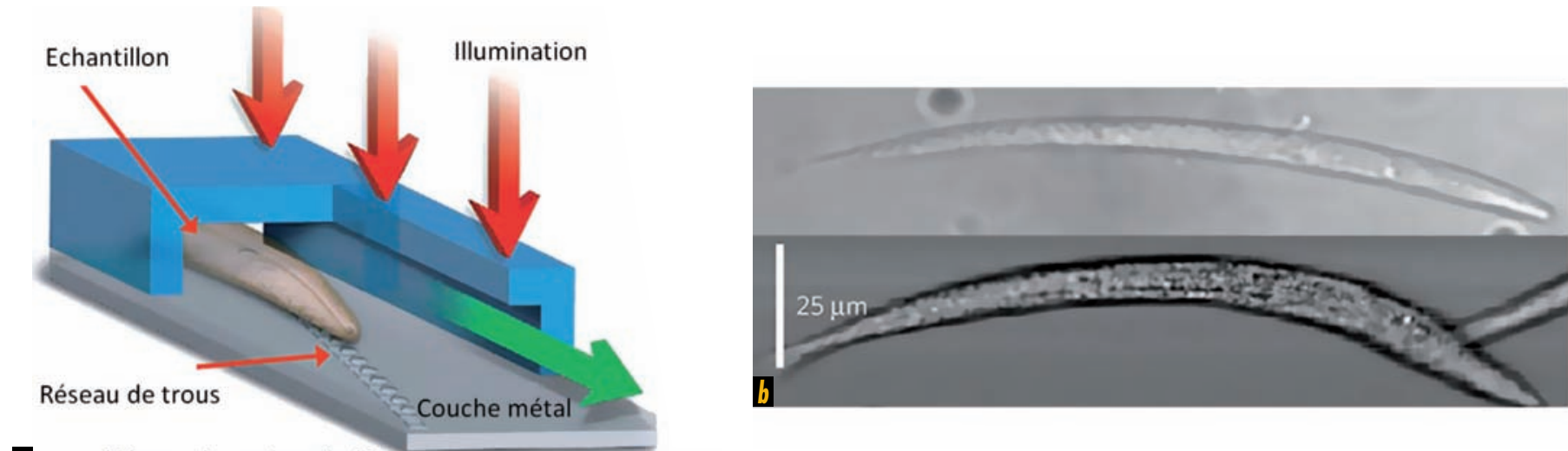

a Réseau de capteur linéaire

Direction de flux

Figure 5. Microscope optofluidique : (a) principe de fonctionnement et (b) comparaison d'une image de microscope conventionnelle (en haut) et d'une image de microscopie optofluique (en bas) (cf. Psaltis et al., Nature, 2006).

Détecter des biomolécules et des cellules

La détection des biomolécules et des cellules par une puce optofluidique est souvent très avantageuse en raison de sa capacité à contrôler les flux, à fonctionnaliser les surfaces des parois des micro-canaux, et à intégrer des micro ou nano-éléments d'analyse métalliques, magnétiques ou encore diélectriques. Un exemple est leur détection à l'aide des micro-cavités diélectriques de différentes formes (micro-anneaux, micro-disques, cristaux photoniques, etc.) couplées à un guide d'onde et présentant un haut fac- teur de qualité (faibles pertes d'énergie par cycle optique). La détection d'espèces biologiques en surface d'un réseau de nanostructures métalliques (réseau plasmonique) estégalement possible. En effet, l'excitation résonnante (phénomène de plasmon de surface) de

\section{Cammandez dès maintenant cet anurage !!}

B Sur le site internet: wuru.edition-sciences.cam

On remplissant ce ban de cammande

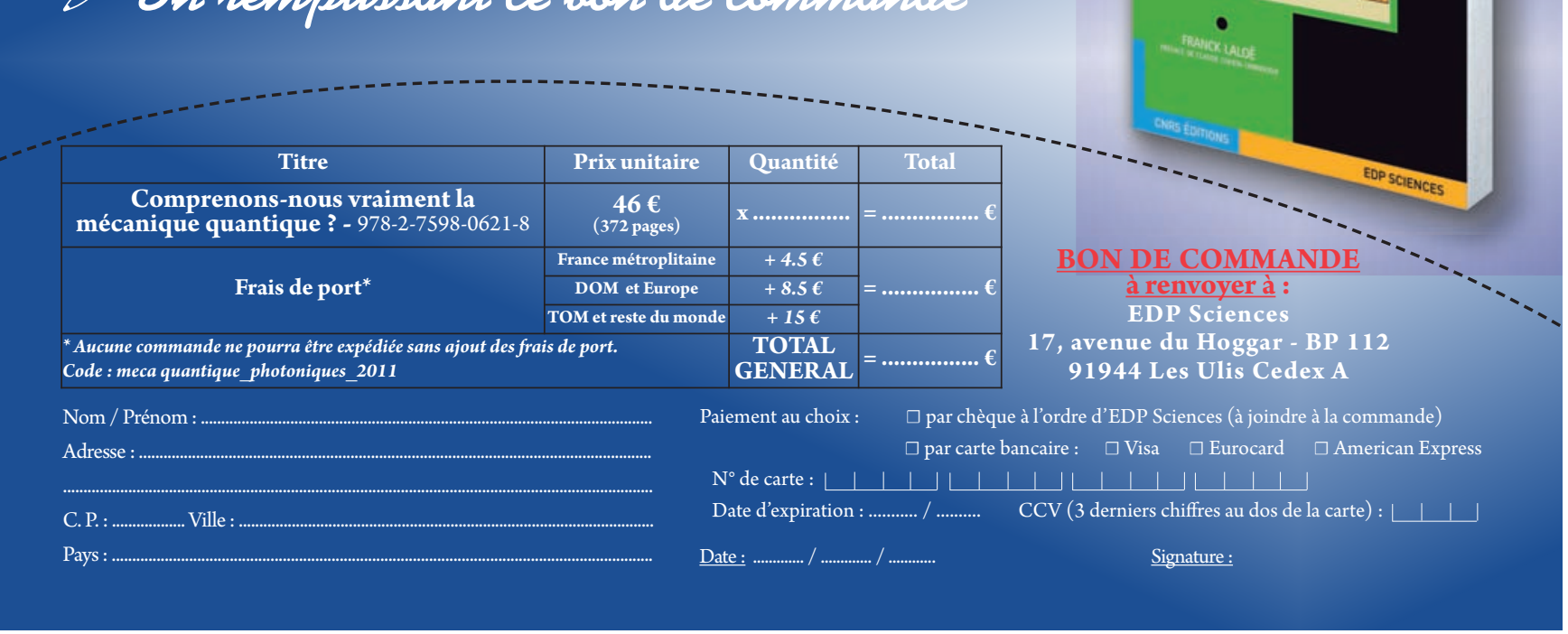


I'oscillation collective des électrons dans une couche métallique est extrêmement sensible au changement d'indice optique au voisinage de la nanostructure. Les puces optofluidiques permettent aussi l'immobilisation puis l'analyse de cellules uniques dans un canal afin de déterminer leurs caractéristiques (type de cellule, état de différenciation, d'apoptoses ou de mitose etc.). Enfin, les puces optofluidiques sont applicables à l'étude de réactions chimiques et biochimiques. Nous avons démontré qu'il était possible de déterminer la concentration d'éthanol d'une solution alcoolisée mélangée avec du potassium dichromate et sulfurique par la mesure du spectre d'absorption de l'échantillon de très faible volume dans une puce optofluidique [2].

\section{Microscopie optofluidique}

Une des très nombreuses applications de l'optofluidique est la microscopie optofluidique dont le développement a reçu une attention particulière. Son principe consiste à laisser passer localement la lumière vers les pixels d'une caméra CCD à travers une couche opaque percée de trous submicroniques alignés sur une diagonale. Une image haute résolution d'une zone très petite d'un objet circulant au-dessus, dans le flux laminaire d'un canal micro-fluidique peut être ainsi obtenue. La figure 5 montre un exemple de cette approche convenant aux objets longs et fins comme le ver Caenorhabditis elegans.

\section{Sources lumineuses optofluidiques}

Naturellement, il est aussi possible de réaliser des sources lumineuses optofluidiques. Dès 2003, plusieurs groupes se sont investis dans la réalisation de microlasers à colorant. Le groupe de recherche danois dirigé par A. Kristensen a proposé un premier dispositif où la cavité laser consiste en deux plaques de verre métallisées placées face à face et entre lesquelles un canal microfluidique a été dessiné. Pompé par un laser Nd:YAG à $532 \mathrm{~nm}$, ce micro-laser à colorant donne une émission laser à $570 \mathrm{~nm}$. En même temps, nous avons proposé, sur le même principe mais en configuration plus intégrable, une cavité laser formée par deux fibres optiques métallisées sur la tranche placées dans le plan de puce et perpendiculaire au canal (figure 6a). L'extraction de l'émission laser est alors assurée par une des fibres. L'accordabilité en longueur d'onde sur une gamme d'une dizaine de nanomètres de ce type de laser a été possible en changeant la concentration du colorant dans une solution d'éthanol. Suite à ce travail, nous avons aussi étudié plusieurs autres types de configurations telles que des microlasers colinéaires à deux longueurs d'onde, des lasers à cavités en anneau ou formés par deux réseaux de Bragg, etc. [3].

En ajoutant un canal d'analyse en intracavité du laser optofluidique, des mesures d'absorption intra-cavité permettent une détection ultra-sensible d'analytes faiblement concentrés [4]. En effet, un petit changement d'absorption, de diffusion ou d'indice de réfraction à l'intérieur ou à la surface de la cavité laser provoque un
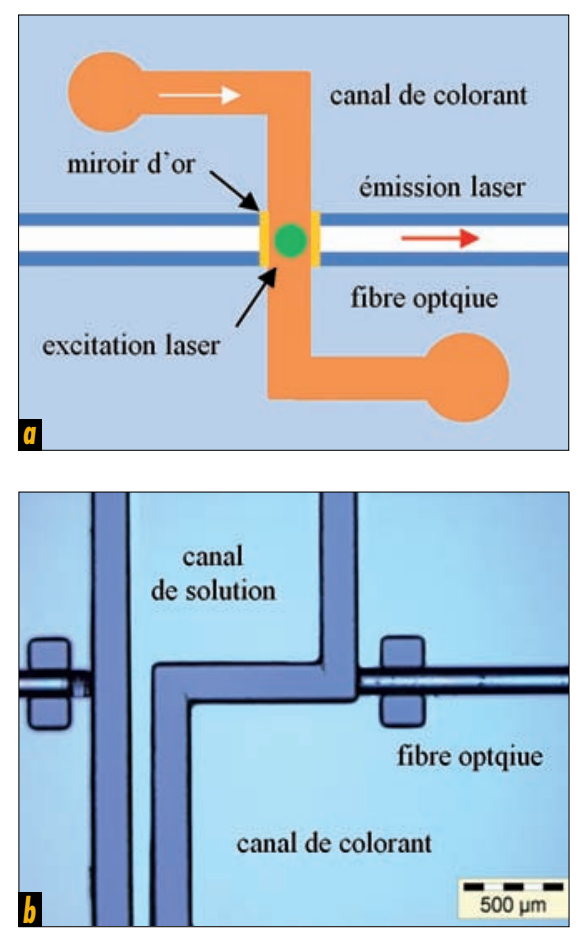

Figure 6. Micro-lasers à colorant : (a) schéma de principe montrant un canal de colorant couplé à deux fibres métallisées sur la tranche et placées face à face pour former une cavité plan-plan et (b) microphotographie d'un dispositif optofluidique pour détecter des molécules à très faible concentration par mesure en intracavité laser. grand changement de l'émission laser à cause de l'effet d'amplification. Nous avons démontré la possibilité de détecter dans un volume de quelques picolitres des molécules dissoutes à une concentration de $10^{-6} \mathrm{~mol}$ par litre (figure $6 \mathrm{~b}$ ). Enfin, I'utilisation des microcavités laser pour l'analyse cellulaire présente aussi un intérêt majeur, puisqu'elle permet de diagnostiquer non seulement l'indice de réfraction de la cellule, mais aussi les organes à l'intérieur de la cellule d'après les nombreuses informations fournies par les modes transverses du système.

\section{Conclusion}

L'association de l'optique à la microfluidique est une approche naturelle et nécessaire. Elle a permis d'exploiter les avantages offerts par la plateforme microfluidique pour la photonique et a aussi contribué de façon significative au progrès de la microfluidique. L'intérêt des puces optofluidiques repose essentiellement sur la faculté de gérer les fluides à faible volume et la capacité d'intégration de différents éléments aux échelles micro et nanométriques.

À cet égard, nous tenons à insister sur l'étendue des possibilités de cette nouvelle branche de recherche pour des applications diverses et variées dans le domaine de l'analyse en chimie, en biologie et biomédicale.

\section{Références}

[1] G. Escalier, Étude de différentes structures optiques passives et actives utilisant et servant la microfluidique, Rapport de Stage Technicien en Optique, LPN/CNRS, Paris, 2002.

[2] L. Lei, Optofluidique : Dispositifs intégrés et applications en physique, chimie et biologie, Thèse de doctorat, Université Pierre et Marie Curie, Paris, 2009

[3] J.G. Calas, Réalisation de lasers microfluidiques à colorant et application à la détection intracavité, Thèse de doctorat, Université Paris-Sud, Orsay, 2006.

[4] Y. Chen et al., Optofluidic microcavities: dye-lasers and bio-sensors, Biomicrofluidics 4, 043002 (2010) 